\title{
The Growth Process of the Soluble Cross-Linked Macromolecule of PU
}

\author{
Fangxing Li*, Ping Jiang, Boyue Wu, Ruimin Sun, Jun Chen, Dongping Liu, Luwei Zhong and \\ Yanfen Wang
}

Department of Chemistry, Nankai University, Tianjin 300071, P.R. China

\begin{abstract}
TEM pictures that were taken at different polymerization times during the formation process of the intramolecularly cross-linked macromolecule (ICM) indicate that ICM growth undergoes three stages. At the first stage, monomers (i.e. prepolymers) react with each other to form small coils, called "dot molecules" (DM), which are shown as small black dots in TEM pictures. At the second phase, the reaction of functional groups on the surface of DMs produces globular intramolecularly cross-linked macromolecules which are called pre-ICMs. At the third stages, the globular preICMs react with each other to form ICMs. As soon as DMs formed, they aggregate into molecular cluster. DMs are restricted within the molecular cluster. This state of aggregation is beneficial to reaction between DMs, since a higher frequency of collision can occur in the molecular cluster. In fact, the molecular cluster is the key polymerization site in the controllable cross-linking polymerization system.
\end{abstract}

Keywords: Controllable cross-linking process, intramolecular reaction, intermolecular reaction, soluble cross-linked macromolecule, pre-ICM.

\section{INTRODUCTION}

The research on the macrogel has expanded remarkably [1-8], with the study of the cross-linking polymerization process remaining the focus of attention [9-12]. However, the polymerization process is still poorly understood. The application of traditional analytical methods has been limited because of the insolubility and infusibility of the macrogel which block experimental study after the gel point has occurred. Computer simulation has extended our understanding of the cross-linking process, including information about the area adjacent to the gel point [13-15]. However, these earlier studies and techniques have not satisfied our desire to understand the mechanisms that control the cross-linking process. It is noteworthy that the gel point can be delayed because of the existence of intramolecular reactions [16-22]. If we increase the amount of intramolecular reaction, the gel point can be increased by more than $100 \%$. This observation indicates that the cross-linking process can be controlled. Therefore, we proposed to find a way to increase the amount of the intramolecular reaction as a means to control the crosslinking process.

In our previous work [23-27], controllable cross-linking systems comprised of intra- and intermolecular reactions were designed based on the macromolecular random coil morphology in solution, in which the ratio of intra- and intermolecular reactions varies over the range of $0 \sim 100 \%$. The molecular weight and the degree of cross-linking can be designed at well. Therefore, such a controllable cross-linking polymerization system is quite different from the uncontrollable ones.

*Address correspondence to this author at the Department of Chemistry, Nankai University, Tianjin 300071, P.R. China;

E-mail: lifangxing @nankai.edu.cn
Tracer experiments revealed that the macromolecules formed in the earliest stages of polymerization have a crosslinked structure and a stable shape because of the intramolecular reactions. These macromolecules, termed dot molecules (DMs) are present as small black dots in TEM pictures. DMs are small in size and have high surface density of active functional groups, which makes them react easily with each other to form bigger molecules. These larger molecules, which we call pre-intramolecularly cross-linked macromolecules (pre-ICMs) in this paper, appear to be $\sim 20$ $30 \mathrm{~nm}$ in diameter in TEM pictures. Two main kinds of reactions can occur between pre-ICMs. (i) The pre-ICMs with few functional residues on the surface are almost incapable of participating in intermolecular reactions and they ultimately, form the smaller ICMs. (ii) The pre-ICMs with more residual groups on the surface can continue to intermolecularly react, forming larger ICMs. Due to the globular structure of the pre-ICMs, the pre-ICMs containing three or more densely functional group areas can form the ICMs with a network structure by reacting with each other; Those preICMs containing two areas of densely packed functional groups can form linear structures, and those containing only a single area of closely located functional groups are the terminating reagents.

Experiments that measured the molecular size found that DMs, pre-ICMs, and ICMs all had an extreme tendancy to form molecular clusters (MCs) in solution. This suggested to us that the cluster is the site of the reaction of DMs and preICMs. We considered that only in the cluster can DMs and pre-ICMs be close enough to each other for reaction, and maintain this close proximity for a long time. Such an environment is conducive to the reaction between DMs and preICMs. Because the surfaces of DMs and pre-ICMs are not smooth, a steric effect may also be involved. Only when they had gathered together closely to smoothly carry out the reaction, or reaction will be extremely slow, even difficult to carry out. 


\section{EXPERIMENTAL SECTION}

\subsection{Materials}

Poly(oxytetramethylene) glycol(PTMG; $M_{n}$ : 1568) was prepared in our laboratory [28]. 4, 4' -diphenylmethane diisocyanate (MDI; Wanhua Co., Yantai PRC), 1,4-butanediol (BD; the First Plant of Chemical Reagents, Tianjin PRC), N,N-dimethyl formamide (DMF; The First Plant of Chemical Reagents, Tianjin PRC) and di-n-butylamine (DBA; The First Plant of Chemical Reagents, Tianjin PRC), were purified by low-pressure distillation prior to use.

\subsection{Polymerization}

The polymerization was carried out in a $500 \mathrm{~mL}$ fourneck flask equipped with a stirring bar. Under an atmosphere of dry nitrogen, MDI was melted in the dry flask. Then PTMG was slowly added dropwise into the flask and maintained at $60{ }^{\circ} \mathrm{C}$ for $75 \mathrm{~min}$. The reaction mixture was then cooled to room temperature and $150 \mathrm{~mL}$ of $\mathrm{DMF}$ and $\mathrm{BD}$ combination were added. The mixture was kept at room temperature for $15 \mathrm{~min}$, and then the reaction temperature was adjusted to $30{ }^{\circ} \mathrm{C}$ and held for $15 \mathrm{~min}$. Then the temperature was further elevated to $60{ }^{\circ} \mathrm{C}$ and kept for an additional $30 \mathrm{~min}$. Then, the temperature was raised to $80{ }^{\circ} \mathrm{C}$ and kept for $385 \mathrm{~min}$. The molar ratio of PTMG: $\mathrm{MDI}$ : $\mathrm{BD}$ in the sample is $1: 2: 0.5$. (According to the main reaction in the paper $[23,25,27]$, the structure of the production is as following:

For the amount of Chain extender(BD) is halved, the number of the -COONH- and the $-\mathrm{N}(\mathrm{CONH}) \mathrm{COO}$ - in each production is equal. We call it Polyurethane-urethaneurea.

Throughout the experimental period, the NCO conversion rate was determined and TEM pictures were taken at the times indicated in Fig. (1).

The number in the box represents the reaction time ( $\mathrm{min}$ ). D: determination of the conversion of NCO by titration; T, termination of the reaction of a sample of polymerization solution $(0.5 \mathrm{~g})$ using $5 \mathrm{~mL}$ DMF and two drops ethylenediamine, and then TEM pictures are taken of a $3 \times 10^{-6}$ $\mathrm{g} / \mathrm{mL}$ solution.

\subsection{Titration}

\subsubsection{The Determination of NCO Conversion}

The NCO functional group reacts with DBA to form urea. After the addition of an aliquot of DBA to the reaction mixture, excess, unreacted DBA is quantitated by titration using $\mathrm{HCl}$. The solvent in all solutions was DMF.

\subsubsection{Preparation of DBA Solution}

DBA (129 g) was added into a $500 \mathrm{~mL}$ brown glass volumetric flask, and then adjusted to volume using DMF.

\subsubsection{Determination Process}

An aliquot (1-12 g) of the reaction mixture was added into a $250 \mathrm{~mL}$ conical flask. (In order to precisely titrate the unreacted DBA, the sampling amount was increased during successive samplings, up to about $12 \mathrm{~g}$ at the final samplings.) The $2 \mathrm{M}$ DBA -DMF solution $(10-30 \mathrm{~mL})$ was added. (As the amount of the later samples was greater, a larger volume of DBA-DMF solution was needed to provide an excess of the reagent.) The mixture was shaken to mix and kept at room temperature for $30 \mathrm{~min}$. An aliquot of the indicator methyl red was added $(0.05 \mathrm{~mL}$ of a $0.1 \%$ methyl red solution prepared in Methanol). The mixture was titrated with $0.5000 \mathrm{~mol} / \mathrm{L}$ of $\mathrm{HCl}$ standard solution until the yellow color changes to red.

The calculation of the NCO conversion is shown as follows:

$P_{N C O}=\frac{\left(V_{0}-V_{s}\right) C \times 125}{1000 m} \times 100 \%=\frac{\left(V_{0}-V_{s}\right) C \times 12.5}{m} \%$

$P_{N C O}$ : the weight fraction of NCO group in reaction mixture.

$V_{0}, V_{s}$ : The volume of $\mathrm{HCl}$ standard solution consumed in blank titration and sample titration, respectively.

$C$ : the concentration of $\mathrm{HCl}$ standard solution. $m$ : sampling mass.

125: half of the molar mass of MDI.

$\eta=1-\frac{P_{N C O} S_{0}}{W_{M D I}}$
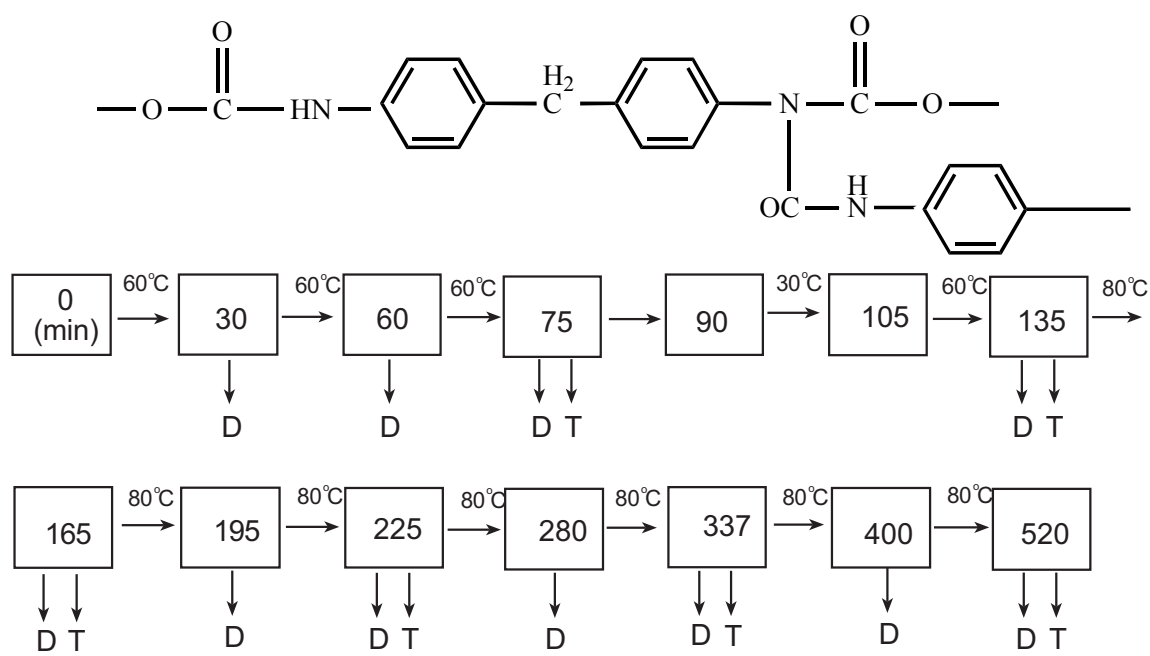

Fig. (1). Schematic representation of the experimental process. 
$\eta$ : NCO conversion.

$S_{0}$ : the mass of reaction mixture at the beginning of the reaction

$W_{M D I}$ : the feed amount of MDI

\subsection{Transmission Electron Microscopy}

The sample of the polymerization solution $\left(3 \times 10^{-6} \mathrm{~g} / \mathrm{mL}\right)$ was placed onto carbon-coated copper grids (400 mesh). The solvent was evaporated in air for $12 \mathrm{~h}$. Electron micrographs were taken on a Philips T20ST transmission electron microscope at an acceleration voltage of $200 \mathrm{kV}$.

\subsection{Molecule Size}

Particle sizes were determined with a photon correlation spectrometer (PCS) using a BI-9000-AT Correlator, a BI200-SM Photometer, and an Innova 304 argon laser (wavelength in a vacuum $\lambda=530.0 \mathrm{~nm}$ ). The system was operated at a scattering angle of $90^{\circ}$, a laser intensity $100 \mathrm{~mW}$, and a temperature of $298 \mathrm{~K}$. The dust in DMF solvent was removed by $0.2 \mu \mathrm{m}$ micropore filter, a $1 \%$ sample solution was prepared with the DMF, and after it was stored for a week, the supernatant solution was used to prepare solutions of other concentrations (solutions No. 1, 2, 3, and 4).Finally, the solution purity was measured with laser light, and it was suitable for molecule size determination by PCS. Three measurements were made for each solution, and the particle sizes are reported in $\mathrm{nm}$.

\section{RESULTS AND DISCUSSION}

\subsection{Synthesis of ICMs}

\subsubsection{Polymerization Process}

The main reactions are shown in Scheme $\mathbf{I}$.

The oligomer capped with NCO is formed by reaction 1 . The chain extension step, shown in reaction 2 , reduces the amount of the chain extender and leads to the formation of NCO-terminated prepolymers [25]. The NCO groups can react with the $\mathrm{H}$ atoms of the $-\mathrm{NHCOO}$ - groups on the prepolymer chains [29]. Reaction 3 involves an intermolecular reaction between two macromolecules. Reaction 4 depicts an intramolecular reaction occurring within a macromolecule. Reactions 3 and 4 are a pair of competing reactions. In the early stages of polymerization, the coil is relatively small with numerous active end-groups on the coil surface, resulting in a high proportion of intermolecular reactions. As the polymerization proceeds, the coil enlarges, a lot of endgroups are consumed and more end-groups are wrapped inside the coil. In the later stages of the polymerization, the intramolecular reaction is favored over the intermolecular reaction, no gelation occurs in the polymerization system and the soluble cross-linked macromolecule is formed.

\subsubsection{The NCO Conversion with Time}

The conversion of NCO groups was determined as a function of polymerization time (Fig. 2). By $135 \mathrm{~min}$, the $\mathrm{NCO}$ conversion reached $76.3 \%$, the most rapid rate of conversion in the whole polymerization process. In this period, reaction 1 and 2 predominate, with the concentrations of $\mathrm{OH}$ and NCO groups being at their highest in the whole polymerization process, resulting in the fastest consumption of NCO. From $135 \mathrm{~min}$, a slower rate of NCO conversion is observed that continues for the remainder of the experimental period. This slow rate of consumption of NCO can be attributed to two factors: 1) the almost complete disappearance of $\mathrm{OH}$ groups from the polymerization system at this time and 2) the occurrence of reactions 3 and 4 in the polymerization system. Reactions 3 and 4 occur mainly between $\mathrm{NCO}$ and the active $\mathrm{H}$ in -NHCOO- group, as well as with the $\mathrm{H}$ in $-\mathrm{OOCN}(\mathrm{R}) \mathrm{CONH}-$ (Scheme I). The velocity of the reactions between $\mathrm{NCO}$ and $\mathrm{OH}$ is faster than that of $\mathrm{NCO}$ with -NHCOO- and -OOCN(R)CONH- [29], so once $\mathrm{OH}$ is
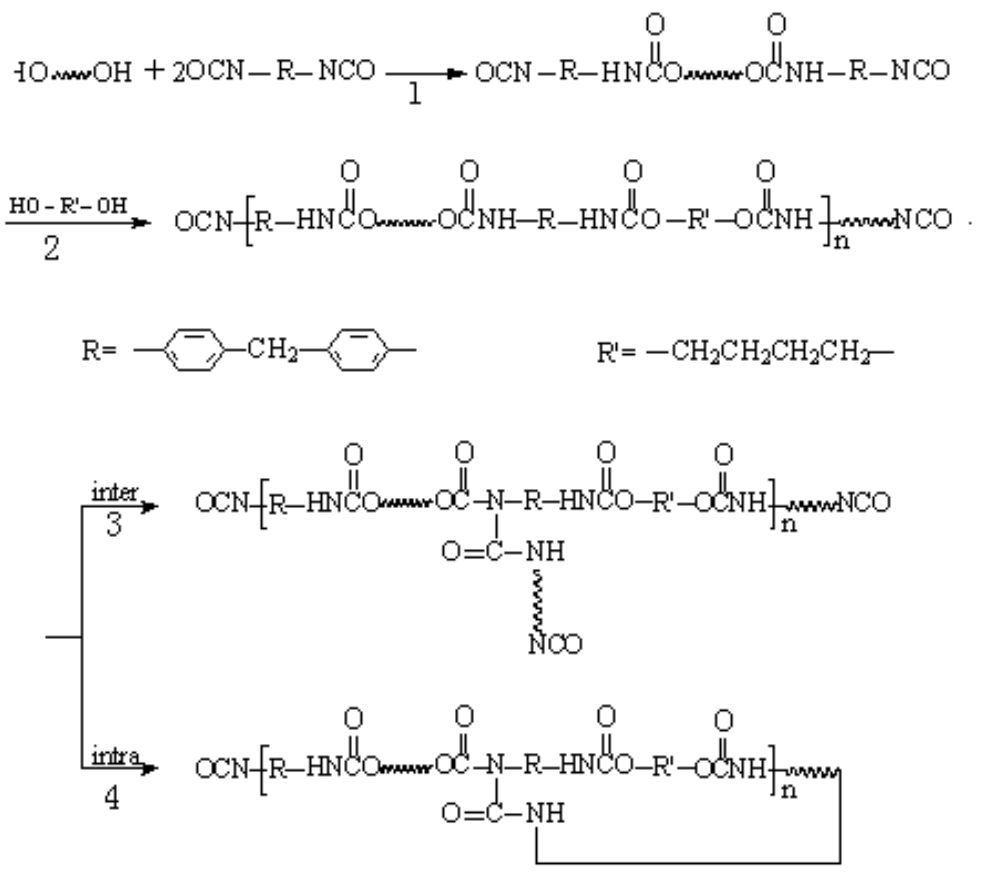

Scheme I. Reactions in the polymerization system. 
consumed, NCO consumption will slow. Therefore, there are big changes in the curve of Fig. (2) at 135 minutes. Because of the low reaction rate of reactions 3 and 4, the conversion does not reach $98 \%$ until 520 minutes.

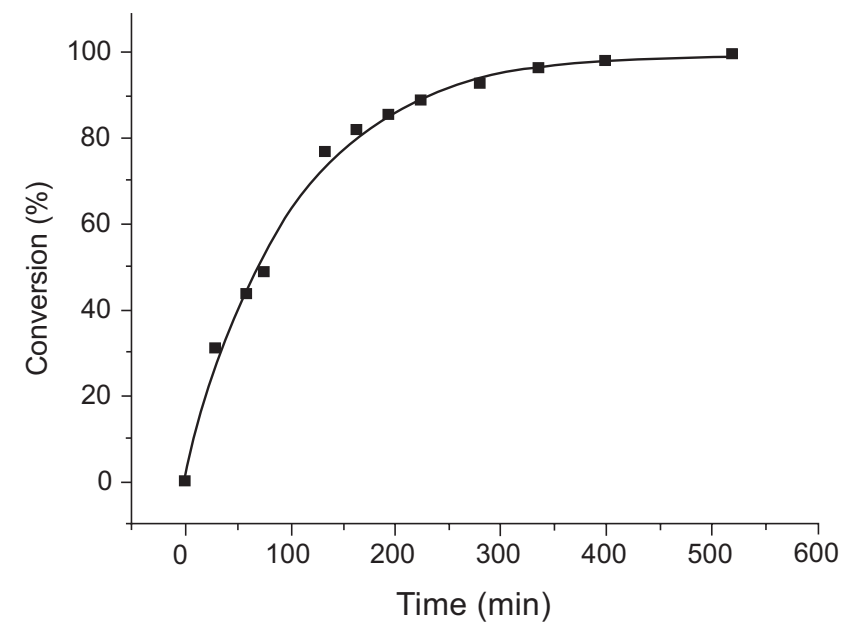

Fig. (2). The NCO conversion with time.

\subsection{The growth process of ICM}

The TEM pictures representing the times in Fig. (1) are shown in Fig. (3).

Graphs 1-1 to $1-4$, taken at 75 minutes as the first reaction has just completed, show small black dots indicating DM. In Graphs 1-2, 1-3, and 1-4, larger particles are also evident that formed during this period. By $135 \mathrm{~min}$, particles larger than DMs but with irregular shapes have formed (Graphs 2-1 to 2-4). In several of the images taken at 135 min, the relatively large particles appear to be composed of many small dots. We have termed these relatively large particles pre-intramolecularly cross-linked macromolecules (pre-ICMs), and the photographs in Graphs 2-1 to 2-4 show the growth process of pre-ICMs. Graph 3-1, taken at 165 min, shows two pre-ICMs with irregular globular shape of different sizes. Graphs 3-2 3-4 document the early formation of intramolecularly cross-linked macromolecules (ICMs) from many smaller globular molecules (pre-ICMs), indicating that ICMs formed step by step. The TEM pictures taken at 225 minutes (Graphs 4-1 to 4-4) and at $337 \mathrm{~min}$ (Graphs 5-1 to 5-4) illustrate the progressive growth of ICMs from smaller macromolecules. As the ICMs get larger and larger, the viscosity of the polymerization system rapidly increases and rod-climbing occurs. At $520 \mathrm{~min}$, DMF (150 $\mathrm{mL}$ ) was added and the reaction was stopped. Over the $3 \mathrm{~h}$ that passed between taking the TEM images in Graphs 5 and 6 , NCO conversion increased by only $3.56 \%$, but the macromolecular size continued to increase. The macromolecules in Graph 6 are the final ICMs which are composed of many globules. Every globule in the ICM derived from a pre-ICM.

\subsection{The Bonds Between Pre-ICM}

Every pre-ICM is large in size and has a very high molecular weight. One single bond is too weak to connect two such globules (Fig. 4).

The tension that two pre-ICMs bear is different, because of the impact of the solvent molecules and the collisions with other pre-ICMs. Therefore, a high concentration of stress occurs at the junction of the pre-ICMs and the single bond will rupture. To connect two globes tightly, multiple bonds are needed, which we have termed "bond clusters" in this paper (Fig. 5). We can find such examples from Graph 3-2, Graph 3-3 and the TEM pictures in ${ }^{[24]}$. In fact, the globules in ICMs in Graph 5 and Graph 6 are connected by bond clusters.

To create bond clusters, each pre-ICM must have several areas of densely spaced surface functional groups (Fig. 5). The bond cluster will form only through the reaction at the areas of densely packed functional groups between two preICMs. In fact, functional groups can not be distributed evenly and the numbers of areas of densely packed functional groups can vary from only one or two to numerous sites on the pre-ICM surface. Fig. (5-1) illustrates three areas of densely spaced functional groups on the surface of the pre-ICM, while Fig. (5-2), shows two areas. Because distribution of the areas of functional groups on the surface of pre-ICMs differ, the final ICM shapes are different.

In the TEM pictures in Graphs 5-1, 5-3, and 6-4 there are pre-ICM dense areas and thin areas. In the dense area, preICM globules form a stacked structure; in the thin area, preICMs connect with each other to form a linear structure. Each pre-ICM needs three or more bond clusters to connect with other globules in the stacked structure. There may be two areas of densely packed functional groups on the surface of globule in the thin pre-ICM area, and hence, only a linear structure can be formed from such globules. Globules having only one area of densely spaced functional groups can become the end group of an ICM. Those globules having no site of dense functional groups will exist independently.

\subsection{The Network Structure in ICM}

The traditional cross-linked structure has been seen as a fishnet, as shown in Fig. (6-A). Until the development of controllable cross-linked polymerization, the process of network formation was poorly understood. Now with new methodology, we can observe the development of the polymerization network.

The TEM photographs (Fig. 3) verify the true structure of the ICM, a most important point. The cross-linked structure in Fig. (6-A) is formed by the reaction of monomers and the distance between cross-linking points is related to the molecular weight of the prepolymers. Such a structure is found in the DM. The images in Fig. (3) show that, once the DMs have formed, the new network can be initiated by further reactions between the DMs (Fig. 6-B). As the DMs have high surface density of functional groups, but very small size, they will form compact cross-linked structures, the pre-ICMs. We can find an example of this structure in Graph 1-4 in Fig. (3). The mesh in Graph 1-4 represents the gap between the DMs. Once the pre-ICM is formed, the third kind of network structure can be produced by the reactions between the pre-ICMs, as shown in Fig. (6-C). These preICMs have areas of functional groups that are either densely packed or are widely distributed. The structure in Fig. (6-A) is defined as the chemical network while that in Fig. (6-C) is defined as the mechanical network. The structure in Fig. (6B) is intermediate between the two networks and is defined as the light mechanical network. Thus, an ICM contains 


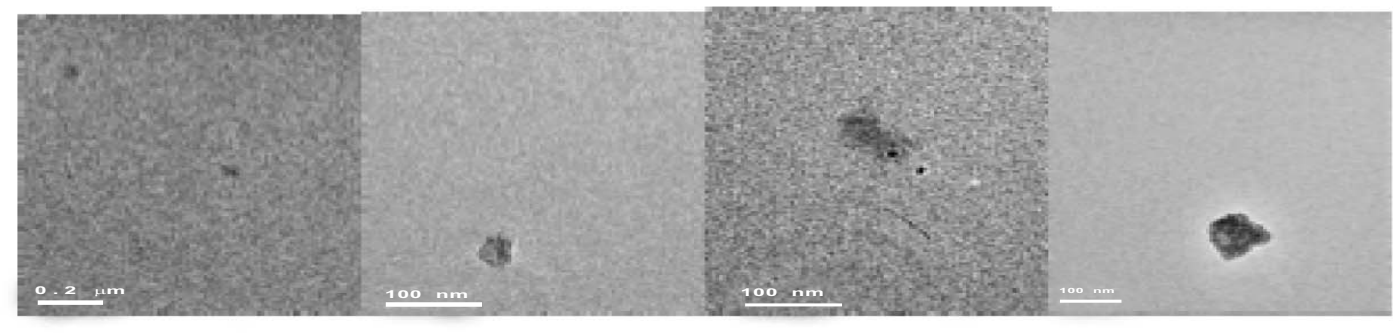

(75 min) $\quad 1-1$

$1-2$

$1-3$

$1-4$
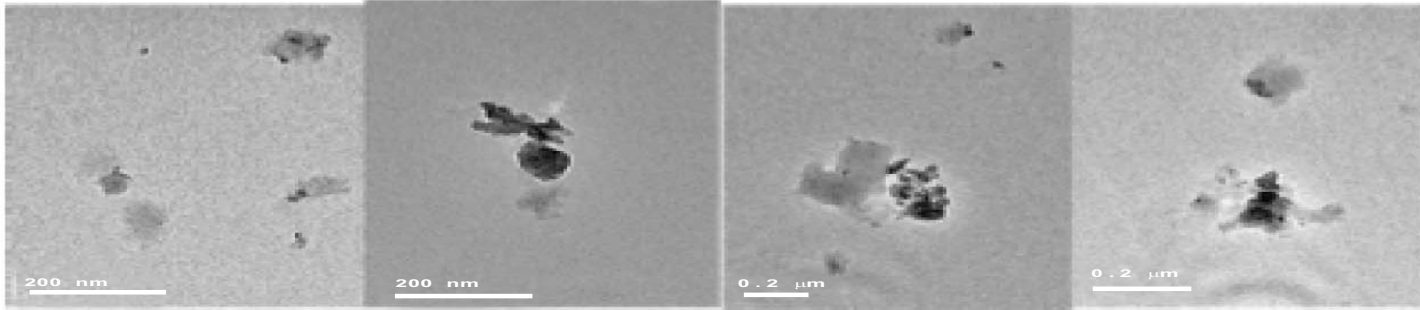

(135 min) 2-1

$2-2$

$2-3$

$2-4$
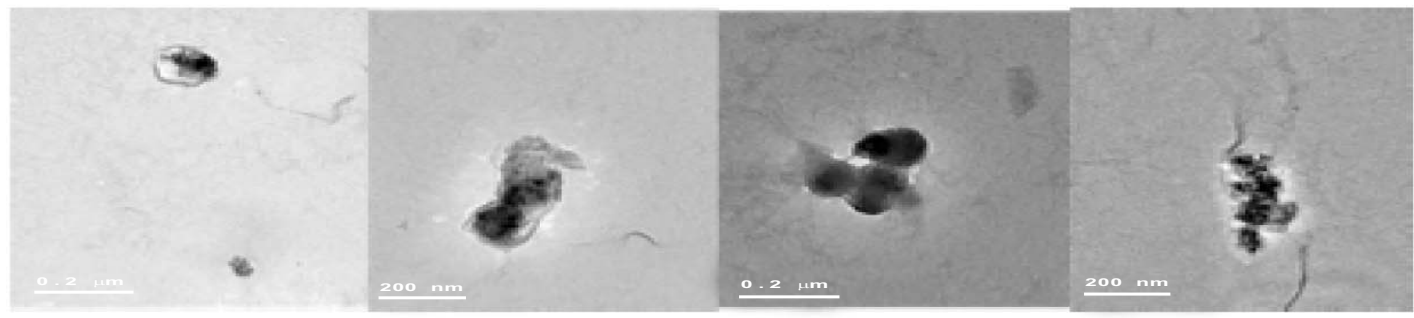

(165 min) 3-1

$3-2$

$3-3$

$3-4$

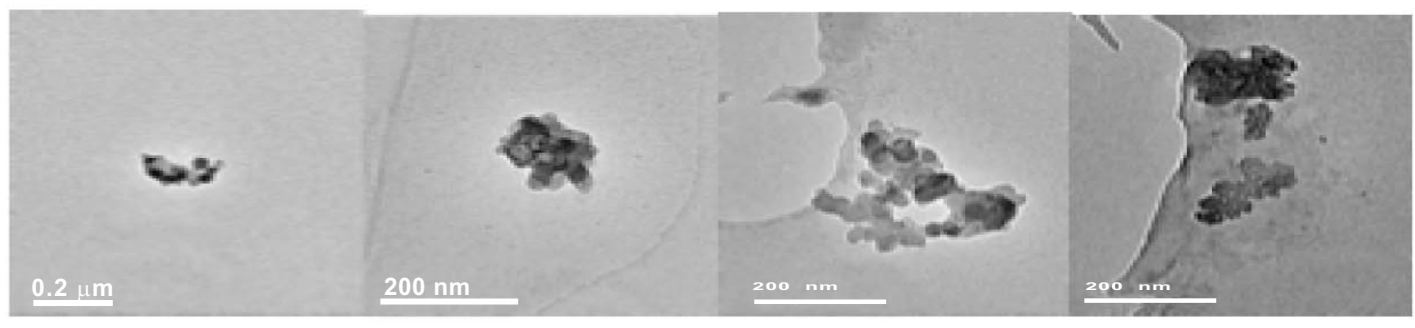

(225 min) 4-1

$4-2$

4-3

$4-4$
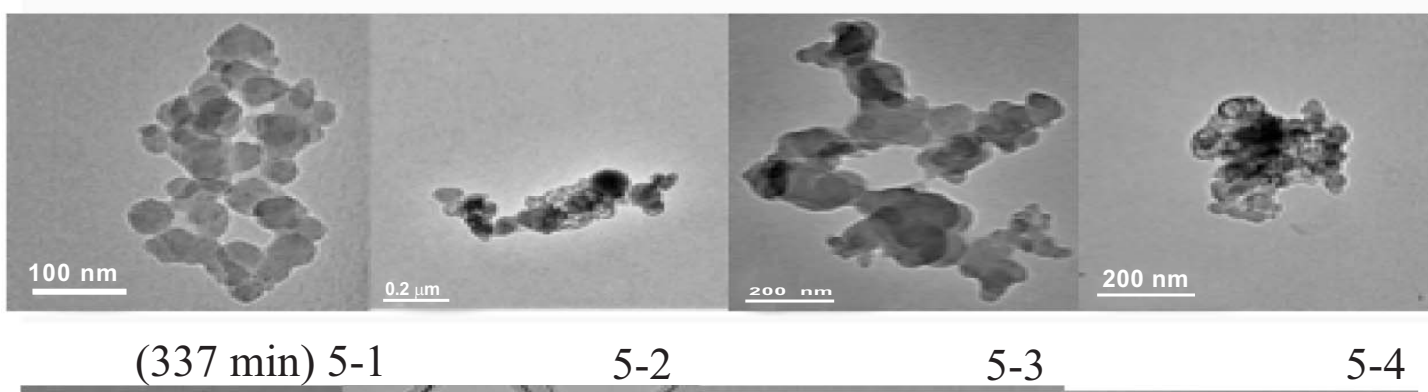

$5-2$

$5-3$

$5-4$
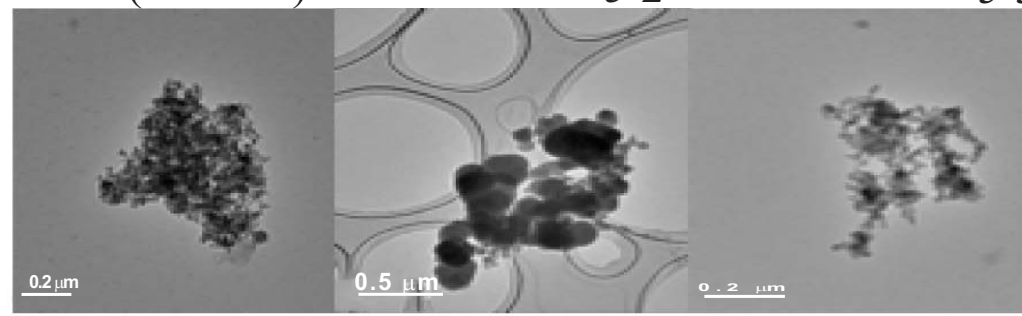

(520 min) 6-1

6-2

6-3

6-4

Fig. (3). TEM pictures of ICM at different reaction times. 


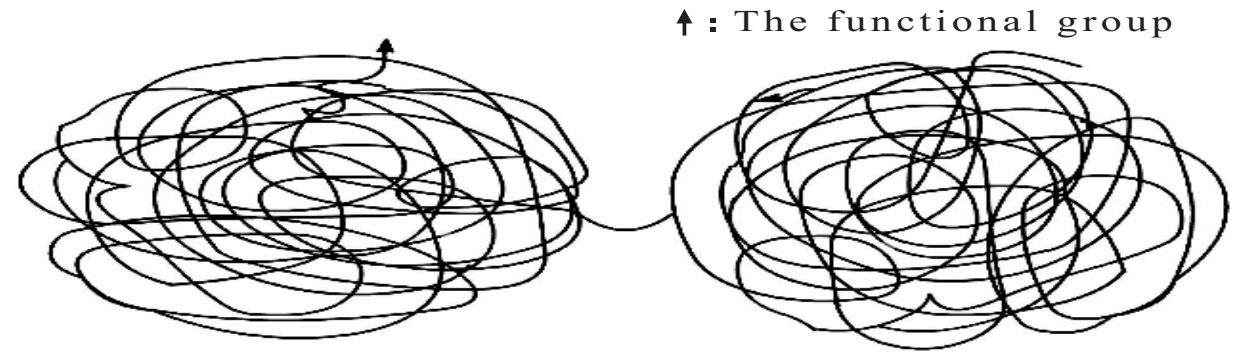

Fig. (4). The schematic representation for two pre-ICMs connected by one single bond.

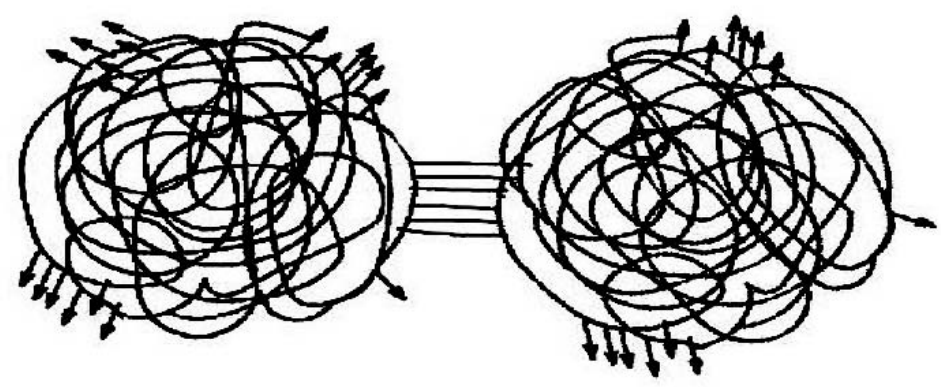

$5-1$

$5-2$

Fig. (5). The schematic representation of pre-ICMs having different amounts of areas of densely populated surface functional groups, connected by a bond cluster.

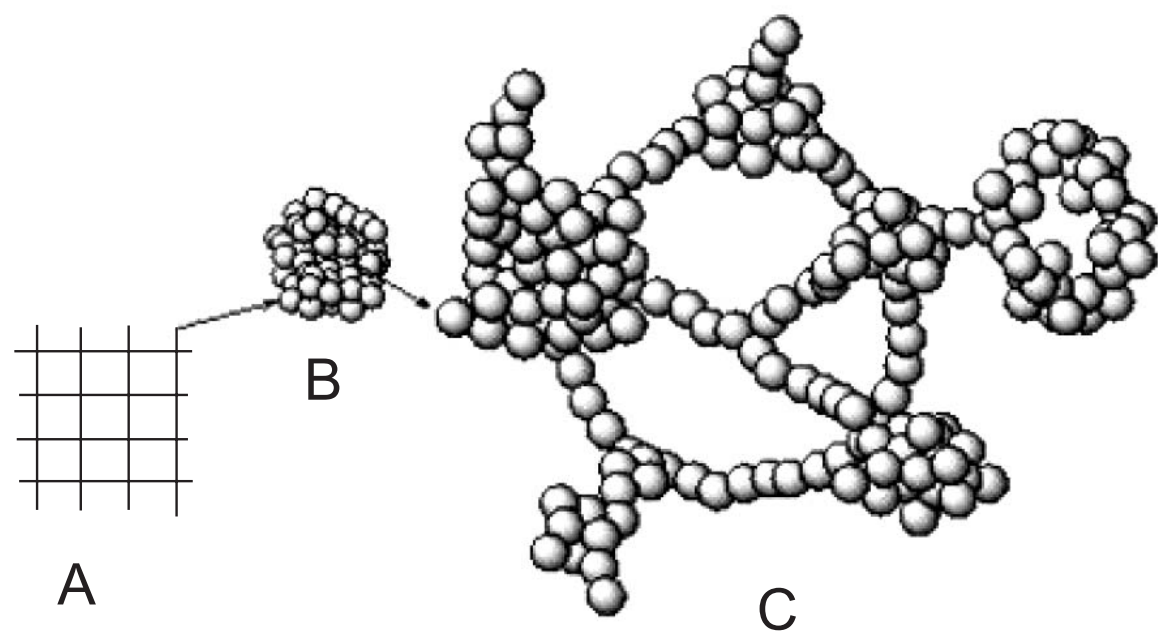

Fig. (6). Three kinds of different network structure in ICM.

A: Chemical network; B: light mechanical network; C: mechanical network.

three types of network structures. In the chemical network, the unit chain is the monomer molecule. In the light mechanical network, the unit chain is a DM. In the mechanical network, the unit chain is a pre-ICM.

\subsection{F ormation of Mo lecular Clu sters in G rowing I CMs Over Time}

\subsubsection{Comparison of the Ability to Form Molecular Clus- ters at $1 \times 10^{-3} \mathrm{~g} / \mathrm{mL}$}

In our previous work, ${ }^{[23-25]}$ ICMs of PU were expected to form molecular clusters in solution, think to its network. In the present study, we wanted to determine the ability of the growing ICMs to form molecular clusters at different times of the polymerization process. We determined molecular size of the particles by PCA measurements and examined the effects of polymerization time and solution concentration on the particle diameters (Tables 1-4). We found that all samples showed a high tendency to form molecular clusters.

In Table 1, the average particle diameter (AE) of $1568 \mathrm{BD}-0.5-75$ is $1712 \mathrm{~nm}$, and corresponds to the DM in Graphs 1 of Fig. (3). This indicates that the DM at the early sampling point of $75 \mathrm{~min}$ has a very strong ability to form molecular clusters. The AE of the sample measured under similar concentration and conditions at $135 \mathrm{~min}$ (1568BD$0.5-135$ ) is $6645 \mathrm{~nm}$ (Table 2). When the extender reaction is finished, the molecules in the system appear like molecules 
Table 1. Effect of Polymerization Time on Particle Diameters of Samples1568BD-0.5-75, 1568BD-0.5-165 and 1568BD-0.5-375

\begin{tabular}{|c|c|c|c|}
\hline No. 1568B & D-0.5-75 & 1568BD-0.5-165 & 1568BD-0.5-375 \\
\hline \hline Concentration(g/ml) & $1.00 \times 10^{-3}$ & $9.98 \times 10^{-4}$ & $9.90 \times 10^{-4}$ \\
& 1503 & 2961 & 16493 \\
Particle Diameter(nm) & 669 & 2683 & 30944 \\
& 2964 & 10539 & 10404 \\
\hline A E $^{\text {a,b }}$ & 1712 & 5394 & 79280 \\
\hline R E $^{c}$ & 2295 & 7856 & 20540 \\
\hline
\end{tabular}

In Tables 1-4, 1568 is the molecular weight of PTMG, BD is the chain extender, 0.5 is the molar ratio of the chain extender $\mathrm{H}$ atom to the NCO group in the oligomer formed in reaction 1 (Scheme I). Times of polymerization ( $\mathrm{min}$ ) are indicated by $75,135,165,225,375$ and 520 . Particle size is determined at a concentration of $\sim 1 \times 10^{-3} \mathrm{~g} / \mathrm{mL}$. ${ }^{\mathrm{a}} \mathrm{In}$ all Tables, $\mathrm{AE}$ and $\mathrm{RE}$ are processed in the same way. ${ }^{\mathrm{b}} \mathrm{AE}$ is the average value of the readings. ${ }^{\mathrm{c}} \mathrm{RE}$ is the range difference between the maximal and the minimal readings.

Table 2. The Effect of Concentration on Particle Diameters of Sample 1568BD-0.5-135

\begin{tabular}{|c|c|c|c|}
\hline No. I-1 & ${ }^{\mathrm{d}} \mathbf{I - 2}$ & $\mathbf{I - 3}$ & $0.95 \times 10^{-5}$ \\
\hline \hline Concentration(g/ml) & $0.95 \times 10^{-3}$ & $0.95 \times 10^{-4}$ & 19060 \\
& 961 & 2337 & 14660 \\
Particle Diameter(nm) & 9962 & 2224 & 7062 \\
& 15155 & 5884 & 16246 \\
\hline A E & 500 & 3308 & 14257 \\
\hline R E & 6645 & 3660 & 11998 \\
\hline
\end{tabular}

${ }^{\mathrm{d}}$ In Tables 2, 3 and 4, the Roman numeral represents the serial number of the sample, and the Arabic number represents the serial number of the solution. Solutions No. 1, 2, 3, and 4 were prepared in DMF as described in the Experimental Section.

Table 3. The Effect of Concentration on Particle Diameters of Sample 1568BD-0.5-225

\begin{tabular}{|c|c|c|c|}
\hline No. & I-1 I-2 & I-3 & $1.0 \times 10^{-5}$ \\
\hline \hline Concentration(g/ml) & $1.0 \times 10^{-3}$ & $1.0 \times 10^{-4}$ & 13927 \\
\hline & 13845 & 2535 & 16761 \\
Particle Diameter(nm) & 3004 & 2279 & 10522 \\
& 3873 & 3463 & 16124 \\
\hline A E & 38971 & 3347 & 14322 \\
\hline R E & 6362 & 1184 & 5602 \\
\hline
\end{tabular}

Table 4. The Effect of Concentration on Particle Diameters of Sample 1568BD-0.5-520

\begin{tabular}{|c|c|c|c|c|}
\hline No. & I-1 I-2 & I-3 & I-4 & $0.95 \times 10^{-5}$ \\
\hline \hline Concentration(g/ml) & $0.95 \times 10^{-3}$ & $0.95 \times 10^{-4}$ & 16516 & 6009 \\
\hline & 6116 & 6910 & 14166 & 13831 \\
Particle Diameter(nm) & 8010 & 11320 & 11969 & 22333 \\
& 22995 & 1899 & 23457 & 165036 \\
\hline A E & 12374 & 5211 & 16527 & 17439 \\
\hline R E & 16879 & 10605 & 1488 & 17027 \\
\hline
\end{tabular}


in Graphs 2 and 3 of Fig. (3) which are pre-ICMs. Obviously the molecular clusters formed by pre-ICMs are bigger then those formed by DMs, indicating that the ability to form molecular cluster of the pre-ICM is stronger than that of the DM. Below we will address the question as to why there is a single kind of network structure in a DM, but two kinds of structures in the pre-ICM.

In Table 4, the AE of $1568 \mathrm{BD}-0.5-520$ at $1 \times 10^{-3} \mathrm{~g} / \mathrm{mL}$ reached $12374 \mathrm{~nm}$. At this time, the molecules in solution are almost all ICMs, and obviously the size of the molecular cluster formed by ICMs is much bigger than that of the preICMs, indicating that the ability to form molecular clusters of the ICM is exceeds that of the pre-ICM. Obviously, there are three kinds of network structure in an ICM.

\subsubsection{The Contributions of Networks to Form Molecular Clusters}

In Tables $\mathbf{2}$ and $\mathbf{3}$, the cluster sizes are very similar even though the solution concentrations are very different. Consistent with this observation are the TEM photographs in Graph 2 and Graph 3 of Fig. (3) which show very similar molecular structures. As the polymerization process proceeds to 520 min, both the TEM photographs in Graph 6 of Fig. (3) and the data in Table 4 indicate the increased size of the molecular structures. The molecules in sample 1568BD-0.5-520 contain all three types of network structures which leads to the differences in cluster size. With the passage of time, the molecular weight and molecular size increase; intramolecular reactions occur more frequently, and the degree of crosslinking would increase. The biting ability of the ICMs would also increase, and the ability to form molecular clusters rises [24].

It is worth pointing out is that in Tables $\mathbf{2}, \mathbf{3}$, and $\mathbf{4}$, the cluster size in the No.3 solution increased as the concentration decreased. This phenomenon was surprising because the molecular weights of both the 1568-0.5-135 and 1568BD0.5-225 samples are small. The 1568-0.5-135 molecule only has chemical and light mechanical networks (Fig. 6). In the 1568-0.5-225 molecule, the mechanical network has just begun to form, and it is still a minor component of the network structure. Thus, primarily the chemical network, and to a lesser degree, the light mechanical network contribute to this phenomenon. From these preliminary results, we infer that in the No.1 solution, the formation of molecular clusters arises primarily from the contributions of the mechanical networks and the light mechanical networks, rather than from the chemical networks. In No.2 solution, the contributions to form clusters come primarily from the light mechanical networks and mechanical networks, particularly the light mechanical network. In the more dilute No.3 solution, the cluster formation depends particularly on the chemical network and to a lesser degree on the light mechanical network. Finally, in the most dilute No.4 solution, molecular cluster formation arises almost completely from the contribution of the chemical network. Therefore, these results have given us a basis to explain the changes of cluster size that occur as concentration changes.

\subsection{Molecular Cluster \& Polymerization Reaction}

The polymerization system described in this paper has three stages. Reaction first occurs between the small glob- ules after DMs have formed. Small globules are composed of DMs, pre-ICMs, and the intermediate states between them. The process of reaction between the small globules in the polymerization system differs from the reaction that occurs due to collision of function groups of other typical small molecules. First, because the surface of small globules is not fully covered with function group, the collisions between small globules do not always result in collision of reactive functional groups. Secondly, the surface of small globules is not smooth, but contains functional group that introduce steric interferences. These two factors sharply reduce the collision frequency of the functional groups and thus productive reaction is hampered. On the other hand, if the small globules could move independently like the typical small molecule in solution, their rate of motion would be slower than that of a small molecule, and their collision frequency would be slower, too, thus making the rate of reaction very slow. From the curve in Fig. (2), the reaction has a normal rate in spite of the morphology of the small globules. This phenomenon shows that the polymerization system has a special environment which increases the reaction rate of the small globules.

As far as DMs are concerned, they are formed as soon as they can aggregate into a molecular cluster. DMs are restricted within the molecular cluster. This state of aggregation is beneficial to reaction between DMs because DMs can obtain higher frequency of collision within the molecular cluster. Therefore, the ability of DMs to form molecular clusters is the condition that permits DMs to react rapidly. At the same time, the molecular cluster is in a state that alternates freely between formation and dissociation. In this process, the small globules can continuously change their contacting surfaces until they succeed in reacting. On the other hand, in the molecular cluster all the globules are linked by "biting" with each other, as illustrated in Fig. (7), thus the surface of the two small globules can fully contact with each other. If the contact site has more functional groups, it will lead to formation of bond clusters, and the two small globules will be tightly linked.

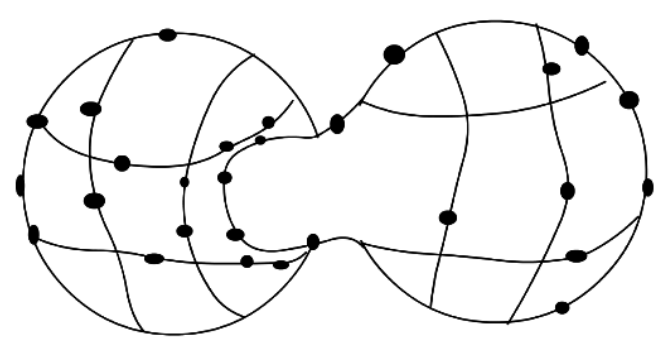

Fig. (7). Full surface contact between the two small globules.

This proposed explanation of the process of controllable cross-linking polymerization is in agreement with our experimental observations.

\section{CONCLUSION}

The growth process of the ICM undergoes three stages in a controllable cross-liking polymerization system. At the first phase, DM forms; at the second phase, pre-ICMs form; at the third phase, ICM forms. Three kinds of networks are formed in these three phases, a chemical network, a light 
mechanical network, and a mechanical network. Pre-ICMs are connected only if bond clusters are formed between two of the small globules.

\section{REFERENCES}

[1] Terashima, T.; Ovchi, M.; Ando, T.; Kamigaito, M.; Sawamoto, M. Metal-complex-bearing star polymers by metal-catalyzed living radical polymerization: Synthesis and characterization of poly(methyl methacrylate) star polymers with Ru(II)-embedded microgel cores. J. Polym. Sci. Part A Polym. Chem., 2006, 44, 4966.

[2] Marcilla, R.; Sanchez-Paniagua, M.; Beatrizlopez-Ruiz, LopezCabarlos, E.; Ochoteco, E.; Grande, H.; Mecerreyes, D. Synthesis and characterization of new polymeric ionic liquid microgels. $J$. Polym. Sci. Part A Polym. Chem., 2006, 44, 3958.

[3] Bouilhach, C.; Cramail, H.; Cloutet, E.; Deffieux, A.; Taton, D. Benzophenone-functionalized, starlike polystyrenes as organic supports for a tridentate bis(imino)pyridinyliron/trimethylaluminum catalytic system for ethylene polymerization. J. Polym. Sci. Part A Polym. Chem., 2006, 44, 6997.

[4] Omari, A.; Tabary, R.; Rousseau, D.; Calderon, L. F.; Monteil, J.; Chauveteau, G. Soft Water-soluble microgel dispersions:Struture and rheology. J. Colloid Interface Sci., 2006, 302, 537.

[5] Hazot, P.; Chapel, I.P.; Pichot, C.; Elaissarl, A.; Delair, T. Preparation of poly(N-ethyl methacrylamide) particles via an emulsion/precipitation process: The role of the crosslinker. J. Polym. Sci. Part A Polym. Chem., 2002, 40, 1808.

[6] Garcia-Salinas, M.J.; Romero-Cano, M.S.; de ias Nieves, F.J. Electrokinnetic Characterization of Poly(N-isopropylacrylamide) $\mathrm{Mi}$ crogel Particles: Effect of Electrolyte Concentration and Temperature. J. Colloid Interface Sci., 2001, 241, 280.

[7] Paulin, S.E.; Bruce J.; Ackerson; Wolfe, M.S. Equilibrium and Shear Induced Nonequilibrium Phase Behavior of PMMA Microgel Spheres. J. Colloid Interface Sci., 1996, 178, 251.

[8] Fukutomi, T.; Oomori, H.; Sucito, Y.B. Connection of poly(methyl methacrylate) microgel particles dispersed in poly(vinyl alcohol) matrix by seed polymerization. J. Polym. Sci. Part A Polym. Chem., 1996, 34, 2729.

[9] Chiou, B.S.; Raghavan, S.R.; Khan, S.A. Effect of Colloidal Fillers on the Cross-Linking of a UV-Curable Polymer: Gel Point Rheology and the Winter-Chambon Criterion. Macromolecules, 2001, 34,4526 .

[10] Takemasa, M.; Chiba, A.; Date, M. Gelation Mechanism of $\kappa$ - and -Carrageenan Investigated by Correlation between the StrainOptical Coefficient and the Dynamic Shear Modulus. Macromolecules, 2001, 34, 7427.

[11] Kakiuchi, M.; Aoki, Y.; Watanabe, H.; Osaki, K. Viscoelastic Properties of Poly(vinyl chloride) Gels: Universality of Gel Elasticity. Macromolecules, 2001, 34, 2987.

[12] Okay, O.; Reddy, S.K.; Bowman, C.N. Molecular Weight Development during Thiol-Ene Photopolymerizations. Macromolecules, 2005, 38, 4501 .

[13] Quintero-Ortega, I.A.; Vivaldo-Lima, E.; Luna-Barcenas, G.; Alvarado, J.F.J.; Louvier-Hernandez, J.F.; Sanchez, I.C. Modeling of the Free-Radical Copolymerization Kinetics with Cross Linking of
Vinyl/Divinyl Monomers in Supercritical Carbon Dioxide. Ind. Eng. Chem. Res., 2005, 44, 2823.

[14] Lamm, M.H.; Chen, T.; Glotzer, S.C. Simulated Assembly of Nanostructured Organic/Inorganic Networks. Nano Lett., 2003, 3, 989.

[15] Lang, M.; Goritz, D.; Kreitmeier, S. Intramolecular Reactions in Randomly End-Linked Polymer Networks and Linear (Co)polymerizations. Macromolecules, 2005, 38, 2515.

[16] Pereda, S.; Brandolin, A.; Valles, E.M.; Sarmoria, C. Copolymerization between $\mathrm{A}_{3}$ and $\mathrm{B}_{2}$ with Ring Formation and Different Intrinsic Reactivity in One of the Monomers. Macromolecules, 2001 34, 4390 .

[17] Gordon, M.; Temple, W.B. The Graph-Liks State of Molecules (III. Ring-Chain C0mpetition Kineties in a Linear Polymerization Reaction). Makromol. Chem., 1972, 160, 263.

[18] Temple, W.B. The Graph-Liks State of Matter (IV. Ring-Chain Competition Kineties in a Branehed Polymerization). Makromol. Chem., 1972, 160, 277.

[19] Leung, Y.; Eichinger, B.E. Computer simulation of end-linked elastomers. I. Trifunctional networks cured in the bulk. J. Chem Phys., 1984, 80, 3877.

[20] Leung, Y.; Eichinger, B.E. Computer simulation of end-linked elastomers. II. Bulk cured tetrafunctional networks. J. Chem. Phys., 1984, 80, 3885.

[21] Dutton, S.; Stepto, R.F.T.; Taylor, D.J.R. Angew. Monte-carlo modelling of the formation, structure and properties of polymer networks. Makromol. Chem., 1996, 240, 39.

[22] Rolfes, H.; Stepto, R.F.T. Monte Carlo modelling of gel points and final network modulus. Polym. Gels Netw., 1994, 2, 149.

[23] Li, F.X.; Liu, Z.F.; Qian, H.T.; Rui, J.M.; Chen, S.N.; Jiang, P.; An, Y.L.; Mi, H.F. Preparation and Size Determination of Soluble Cross-Linked Macromolecule of Polyurethane. Macromolecules, 2004, 37, 764.

[24] Li, F.X.; Liu, Z F.; Liu, X.P.; Yang, X.Y.; Chen, S.N.; An, Y. L; Zuo, J.; He, B.L. Preparation and Size Determination of a Soluble Cross-Linked Macromolecule of Polyurethane with an Ethylene Diamine Chain Extender. Macromolecules, 2005, 38, 69

[25] Li, F.; Zuo, J.; Dong, L.M.; Wang, H.J.; Luo, J.Z.; Han, W.; Huo, Y. Study on the Synthesis of High Elongation Polyurethane. Eur. Polym. J., 1998, 34, 59.

[26] Li, F.; Ju, Z.; Dong, L.; Zhou, Q.; Han, W.; Luo J.Z.; Han, W.; Huo, Y. The study of synthesis of poluurethane by extending chain of monoacidic amine. Polym. Mater. Sci. Eng., 1999, 15, 55.

[27] Liu, Z.; Wu, X.; Yang, X.; Liu, D.; Jun, C.; Sun, R.; Liu, X.; Li, F. Synthesis and Characterization of Novel Blood-Compatible Soluble Chemically Cross-Linked Polyurethanes with Excellent Mechanical Performance for Biomedical Applications. Biomacromolecules, 2005, 6,1713

[28] Li, F.X.; Wang, H.J.; Li, C.G.; Ma, K.Q. Study on Chain Transfer to Acetic Anhydride of THF Polymerization. J. Polym. Sci. A. Polym. Chem., 1994, 52, 1939.

[29] Lyman, D.J. The Chemistry of the Diisocyanate-Diol reaction. Solomon, D. H., Ed. In Step-Growth Polymerizations, $2^{\text {nd }}$ ed., Marcel Dekker Inc: New York; 1972, p. 98.

(C) Li et al.; Licensee Bentham Open.

This is an open access article licensed under the terms of the Creative Commons Attribution Non-Commercial License (http://creativecommons.org/licenses/by-nc/3.0/) which permits unrestricted, non-commercial use, distribution and reproduction in any medium, provided the work is properly cited. 